\title{
Computer-Assisted Volumetric Measurement of Core Infarct Volume in Pediatric Patients: Feasibility for Clinical Use and Development of Quantitative Metrics for Outcome Prediction
}

\author{
C.G. Filippi, A.M. El-Ali, V.Z. Miloushev, D.S. Chow, X. Guo, and B. Zhao
}

\begin{abstract}
BACKGROUND AND PURPOSE: Infarct volume may predict clinical outcome in acute stroke, but manual segmentation techniques limit its routine use. We hypothesized that computer-assisted volumetric analysis to quantify acute infarct volume will show no difference compared with manual segmentation but will show increased speed of performance and will correlate with outcome.
\end{abstract}

MATERIALS AND METHODS: Patients with acute stroke younger than 18 years were included. Infarct volume on diffusion-weighted imaging was quantified by using computer-assisted volumetric and manual techniques. The Pediatric Stroke Outcome Measure scored clinical outcome. Computer-assisted volumetric and manual techniques were compared with correlation coefficients. Linear regression analysis compared Pediatric Stroke Outcome Measure with core infarct volume and percentage volume of brain infarction.

RESULTS: Twenty-three patients were analyzed (mean age, 4.6 years). Mean infarct volume from computer-assisted volumetric and manual approaches was 65.6 and $63.7 \mathrm{~mL}$, respectively $(P=.56)$. Concordance correlation between methods was 0.980 , and between users, 0.968. The mean times for segmentation between computer-assisted volumetric and manual techniques were $<1$ minute and 7.3 minutes $(P<.001)$. The mean infarct volumes for good and poor outcome groups were 7.4 and $75.7 \mathrm{~mL}(P<.007)$. The mean percentages of infarcted brain parenchyma for good and poor outcome groups were $0.6 \%$ and $10.4 \%(P<.006)$. Volumes of $32 \mathrm{~mL}$ and $3 \%$ for infarcted brain were associated with poor outcome in all patients.

CONCLUSIONS: Computer-assisted volumetric quantification of infarct volume is reproducible, is significantly faster than manual techniques, and may have important applications for future clinical workflow. Core infarct volumes and infarct percentage correlated with outcome severity.

ABBREVIATIONS: CAV = computer-assisted volumetry; PSOM = Pediatric Stroke Outcome Measure

S troke in the pediatric population is occurring at increasingly younger ages with an increasing incidence estimated at 3-5 per 100,000 according to the US Nationwide Inpatient Sample (http://www.healthdata.gov/data/dataset/hcup-nationwideinpatient-sample-nis), which showed annual increases in acute ischemic stroke admissions from 1995 to $2008 .{ }^{1-6}$ However, pediatric stroke remains under-recognized among health care providers; a lack of evidence-based treatment and management guidelines specific to

Received June 4, 2014; accepted after revision September 28

From the Department of Radiology, Columbia University Medical Center, New York, New York.

Alexander M. El-Ali, MD, is a recipient of Radiological Society of North America Medical Student Seed Grant 2013-2014.

Paper previously presented as an oral abstract at: Annual Meeting of the American Society of Neuroradiology, May 17-22, 2014; Montreal, Quebec, Canada.

Please address correspondence to C.G. Filippi, MD, Columbia University Medical Center, Department of Radiology, 177 Fort Washington Ave, MHB 3-111, New York, NY 10032; e-mail: sairaallapeikko@gmail.com

http://dx.doi.org/10.3174/ajnr.A4183 the pediatric population further complicates this problem. ${ }^{7,8}$ This represents a critical health care problem, given the potential cost to society in terms of life-years of disability and life-years lost in the face of increasing incidence of acute ischemic arterial infarction of children. ${ }^{6,7,9,10}$

Recent studies support infarct volume quantification as a potential tool in the pediatric population for predicting clinical outcome. ${ }^{11-13}$ For example, Ganesan et al ${ }^{14}$ observed that infarcts of $>10 \%$ parenchymal volume on T2-weighted imaging were associated with poor outcomes. Additionally, Domi et $\mathrm{al}^{15}$ reported that reduced diffusion in the corticospinal tract was a predictor of motor outcomes. Furthermore, investigations in adults have suggested that core infarct volume quantification correlates best with long-term outcome; this finding lends credibility to a similar approach in pediatric stroke. ${ }^{16-19}$

Currently, the criterion standard for volumetric assessment involves manual segmentation, which can be time-consuming and technically challenging. These impediments may limit its use 
in the emergency setting, where time is critical for management. For these reasons, computer-assisted volumetry (CAV) applied to diffusion-weighted imaging may represent a potential tool to aid in the detection of core infarct volume in the pediatric population. With regard to neuroimaging, CAV has recently been used in the examination of recurrent glioblastoma with high reproducibility and speed compared with conventional manual approaches.

The purpose of this study was to describe a novel CAV technique for assessment of core infarct volume within the pediatric population. Specifically, we describe the reliability and feasibility of this technique compared with traditional manual approaches in patients with acute stroke. Additionally, the relationship between infarct volume and clinical outcomes by using the Pediatric Stroke Outcome Measure (PSOM) scale will be obtained. ${ }^{20,21}$

\section{MATERIALS AND METHODS Subjects}

After institutional review board approval of this Health Insurance Portability and Accountability Act-compliant retrospective study, a query of the neuroradiology department data base for pediatric patients with acute arterial ischemic infarct at our institution from January 2011 to November 2012 was conducted. Acute arterial ischemic infarct was defined as an acute neurologic deficit in a patient with an MR imaging abnormality on DWI consistent with infarction and corresponding hypointense signal on the apparent diffusion coefficient map. Inclusion criteria were pediatric patients defined as younger than 18 years of age. Imaging inclusion criteria were MR imaging with DWI performed within 72 hours of admission to the hospital. Exclusion criteria were hypoxic-ischemic encephalopathy, intraparenchymal hemorrhage, prior infarcts, incomplete imaging, or poor image quality (motion degradation) and incomplete followup. Additionally, patients without medical records sufficient to obtain PSOM scores were also excluded.

\section{Imaging Techniques}

All imaging was performed on a 1.5T MR imaging system (Signa; GE Healthcare, Milwaukee, Wisconsin) with an 8-channel headarray coil (Signa HDxt; GE Healthcare). We performed the following sequences: sagittal T1-weighted (TR, $450 \mathrm{~ms} / \mathrm{TE}, 6.5 \mathrm{~ms}$; 5-mm thickness; echo-train length, 4; FOV, $24 \mathrm{~cm} ; 256 \times 224$ matrix); axial T1-weighted (TR, $433 \mathrm{~ms} / \mathrm{TE} 6.5 \mathrm{~ms}$; 5-mm thickness; echo-train length, 4; FOV, $24 \mathrm{~cm}$; $256 \times 224$ matrix); axial T2-weighted (TR, $5000 \mathrm{~ms} / \mathrm{TE}, 90 \mathrm{~ms}$; 5-mm thickness; echotrain length, 28; FOV, $24 \mathrm{~cm} ; 320 \times 320$ matrix); and axial FLAIR imaging (TR, $8800 \mathrm{~ms} / \mathrm{TE}, 120 \mathrm{~ms}$; TI, $1650 \mathrm{~ms}$; echo-train length, 1.0; 5-mm thickness; FOV, 24 cm; $256 \times 192$ matrix). Axial diffusion-weighted imaging (TR, $8000 \mathrm{~ms} / \mathrm{TE} 88 \mathrm{~ms}$; echo-train length, 1.0; 5-mm thickness; $128 \times 128$ matrix; FOV, $24 \mathrm{~cm})$ was performed by using $2 \mathrm{~b}$-values $\left(0\right.$ and $1000 \mathrm{~s} / \mathrm{mm}^{2}$ ) and 3 diffusion-encoding gradient directions. Corresponding ADC maps were generated by commercial scanner software (FuncTool software; GE Healthcare).

\section{Core Infarct Volume Segmentation}

Two radiologists, including 1 with a Certificate of Added Qualification in neuroradiology, performed CAV analysis. The boundary of acute infarct was delineated on DWI sequences by using a

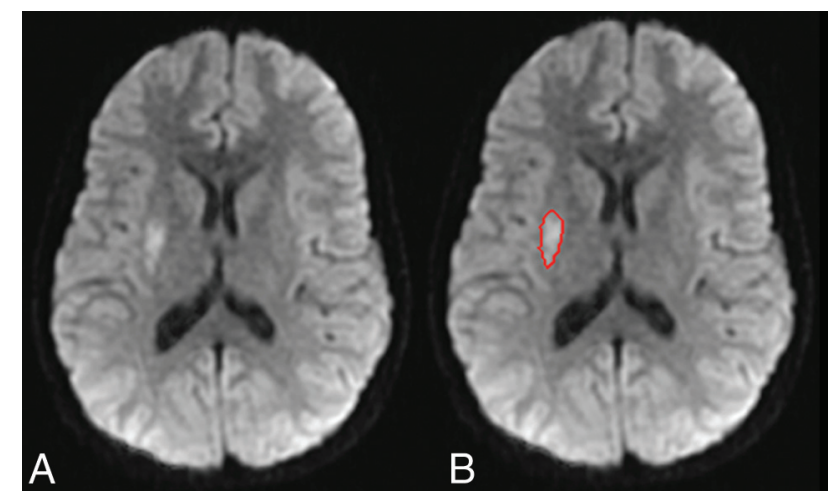

FIG 1. $A$, Reduced diffusivity within the right putamen consistent with an area of acute infarction. $B$, The contour derived from the semiautomated computer segmentation software that is used to derive infarct volume.

Table 1: Demographics of patients

\begin{tabular}{lc}
\hline \multicolumn{1}{c}{ Variable } & No. (\%) \\
\hline Sex & \\
Male & $12(52.2)$ \\
Female & $11(47.8)$ \\
Ethnicity (No., \%) & \\
White & $4(17.4)$ \\
Black & $2(8.7)$ \\
Asian & $0(0.0)$ \\
Hispanic & $2(8.7)$ \\
Other & $15(65.2)$ \\
Stroke risk factors (No., \%) & \\
No risk factors & $5(21.7)$ \\
Single risk factors & $11(47.8)$ \\
Multiple risk factors & $7(30.4)$ \\
Risk factor distribution ${ }^{\text {a }}$ (No., \%) & \\
Hematologic & $9(39.1)$ \\
Vascular & $7(30.4)$ \\
Cardiac & $4(17.4)$ \\
Infection & $3(11.1)$ \\
Ingestion & $1(4.3)$ \\
Metabolic & $1(4.3)$ \\
Therapy (No., \%) & \\
Anticoagulation & $4(17.4)$ \\
Antiplatelet & $9(39.1)$ \\
Anticonvulsant & $14(60.9)$ \\
Surgery & $3(13.0)$ \\
\hline
\end{tabular}

a Patients may have $>1$ risk factor.

proprietary segmentation algorithm developed in the Laboratory for Computational Image Analysis in the Department of Radiology, Columbia University Medical Center. ${ }^{22,23}$ The computerassisted algorithm was originally developed for liver lesions and has since been adapted for different applications, including, most recently, the volume of enhancing tumor in glioblastoma multiforme. ${ }^{24-26}$ For the first step of the volume measurement, the entire infarct volume was separated from surrounding anatomic structures by using a segmentation algorithm that combines the image analysis techniques of active contours and a level set approach. Once the segmentation was completed on an image, the infarct contour was propagated to its neighboring images, serving as an initial region of interest for subsequent segmentations on the neighboring images. This process was continued iteratively until all the infarct images were segmented. Once the segmentation was finalized, infarct volumes were automatically calculated (Fig 1). 
For brain volume, we determined the contour of the brain cortical surface from which ventricular volume is subtracted by contouring the ventricular lining.

The same 2 radiologists, both of whom were blinded to the patients' prior CAV volumes and clinical outcomes, performed manual segmentation of infarct volume. Segmentation was per-

Table 2: Patient outcomes and stroke characteristics

\begin{tabular}{lc}
\hline \multicolumn{1}{c}{ Outcome Measures } & (No.) (\%) \\
\hline Good $^{\text {a }}$ Poor $^{\text {b }}$ & $5(21.7)$ \\
Mortality & $18(78.3)$ \\
Infarct circulation & $5(21.7)$ \\
$\quad$ Anterior circulation only & \\
Posterior circulation only & $20(87.0)$ \\
Both circulations & $0(0)$ \\
Infarct involvement & $3(13.0)$ \\
Bilateral & \\
ACA & $11(47.8)$ \\
MCA & $8(34.8)$ \\
PCA & $23(100.0)$ \\
Basal ganglia & $12(52.2)$ \\
\hline
\end{tabular}

Note:-ACA indicates anterior cerebral artery; PCA, posterior cerebral artery.

a "Good outcome" defined as healthy or mild disability using the Pediatric Stroke

Outcome Measure.

b "Poor outcome" defined as moderate or severe disability using the Pediatric Stroke Outcome Measure or death.

Table 3: Stroke characteristics and patient outcomes

\begin{tabular}{|c|c|c|c|c|c|}
\hline \multirow[b]{2}{*}{ Category } & \multirow[b]{2}{*}{ Mean } & \multirow[b]{2}{*}{ Range } & \multicolumn{2}{|c|}{$\begin{array}{l}95 \% \text { Confidence } \\
\text { Interval }\end{array}$} & \multirow[b]{2}{*}{$\begin{array}{c}\text { Standard } \\
\text { Error }\end{array}$} \\
\hline & & & $\begin{array}{l}\text { Lower } \\
\text { Limit }\end{array}$ & $\begin{array}{l}\text { Upper } \\
\text { Limit }\end{array}$ & \\
\hline Total brain volume (mL) & 936.6 & $392.0-1387.0$ & 815.7 & 1057.5 & 61.67 \\
\hline Stroke volume $(\mathrm{mL})$ & 60.9 & $0.1-229.0$ & 31.7 & 90.0 & 14.88 \\
\hline Stroke volume (\%) & 8.2 & $0.1-48.4$ & 3.6 & 12.9 & 2.35 \\
\hline \multicolumn{6}{|l|}{ Good outcome $\mathrm{a}^{\mathrm{a}}$} \\
\hline Stroke volume (mL) & 7.4 & $0.08-31.63$ & 0.97 & 26.12 & 5.48 \\
\hline Stroke volume (\%) & 0.6 & $0.01-2.64$ & 0.05 & 2.12 & 0.45 \\
\hline \multicolumn{6}{|l|}{ Poor outcome ${ }^{b}$} \\
\hline Stroke volume (mL) & 75.7 & $0.40-229.00$ & 47.28 & 115.43 & 16.80 \\
\hline Stroke volume (\%) & 10.4 & $0.05-48.42$ & 6.35 & 17.57 & 2.71 \\
\hline
\end{tabular}

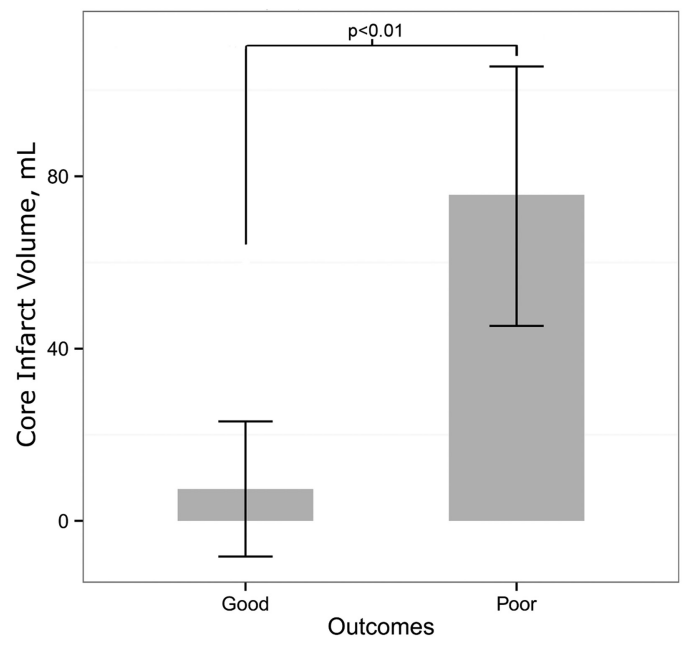

formed by manual tracing of regions of reduced diffusion on DWI sequences. All manual segmentations were performed at a dedicated workstation (Advantage Workstation, Version 4.3; GE Healthcare). To reduce bias from prior CAV analysis, we performed manual measurements 3 months after the initial CAV analysis. All measurements were recorded in milliliters, and the time required to perform CAV and manual segmentation for core infarct volume was noted.

\section{Correlation with Clinical Outcomes}

Clinical outcome was scored by using the PSOM, which uses neurologic evaluation to examine sensorimotor function bilaterally, productive and receptive language, and cognitive and behavioral development. ${ }^{20,21}$ The PSOM is typically scored as good (healthy or mild deficit) and poor (moderate or severe), and validity of the PSOM is not significantly affected when performed retrospectively. ${ }^{20}$ Clinical data and PSOM were determined from chart review by using the examination findings of a pediatric neurologist. All patients were evaluated by a neurology attending physician following stroke, which was confirmed through chart review. Two neuroradiologists performed chart review.

\section{Statistical Analysis}

Mean manual and semiautomated volumetric measurements and segmentation times were compared by using a paired $t$ test. Concordance correlation coefficients with corresponding confidence intervals between semiautomated and manual volumetric measurements were obtained. For analysis of clinical outcomes, core infarct volumes and the percentage of infarcted parenchymal volumes were compared among patients with good and poor outcomes by using the Wilcoxon rank sum test. Linear regression analysis was performed comparing the PSOM with core infarct volume and percentage volume of brain infarction. All statistical analysis was performed by using MedCalc for Windows, Version 12.2.1.0 (MedCalc Software, Mariakerke, Belgium). For this study, a $P$ value of $<.05$ was considered statistically significant.

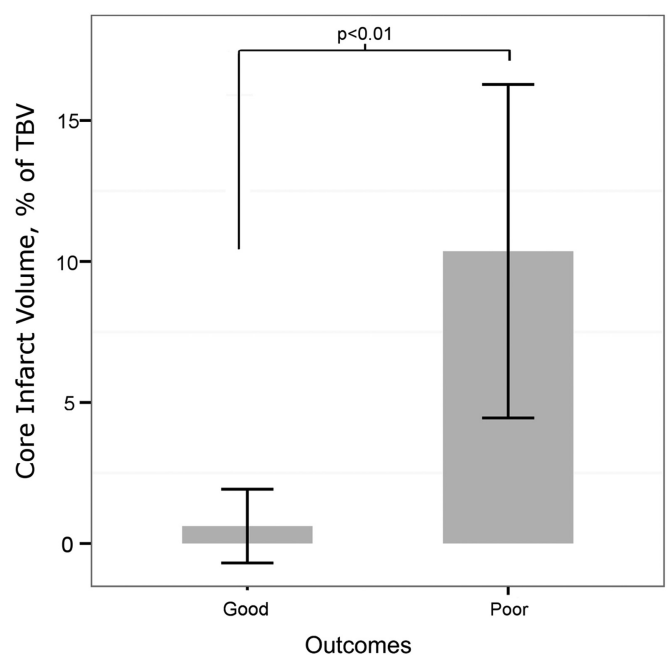

FIG 2. Graphic representation of the correlation of core infarct volume and percentage brain infarction with the Pediatric Stroke Outcome Measure. TBV indicates total brain volume. 


\section{RESULTS}

\section{Subjects}

In total, 29 patients were identified, of which 79.3\% (23/29) met the inclusion criteria. Patient demographics are listed in Table 1. These included 12 male and 11 female patients (age range, $0-17.6$ years; mean age, 4.6 years) presenting with acute stroke. Clinical follow-up ranged from 0.8 to 19.3 months (mean, 5.1 months). Six patients were excluded. One had hypoxic-ischemic encephalopathy rather than acute stroke, 1 had marked intraparenchymal hemorrhage and prior infarcts, 1 had incomplete imaging with poor image quality (motion degradation), and another had incomplete clinical follow-up. An additional 2 patients did not have all test items in their medical records to calculate a PSOM score and were excluded.

\section{Comparison between Manual and CAV Analysis}

The mean core infarct volumes obtained for CAV and manual approaches were 65.6 and $63.7 \mathrm{~mL}$, respectively $(P=.56)$. The mean total brain volume was $936.6 \mathrm{~mL}$. The concordance corre-

Table 4: Infarct volume versus Pediatric Stroke Outcome Measure

\begin{tabular}{lccc}
\hline & $\begin{array}{c}\text { Regression } \\
\text { Coefficient }^{\text {a }}\end{array}$ & $\begin{array}{c}\text { Standard } \\
\text { Error }\end{array}$ & $\boldsymbol{P}$ Value \\
\hline Infarct by percentage & 0.150 & 0.067 & .039 \\
Infarct by absolute volume & 0.028 & 0.010 & .011 \\
\hline
\end{tabular}

${ }^{a}$ Coefficient calculated with linear regression.

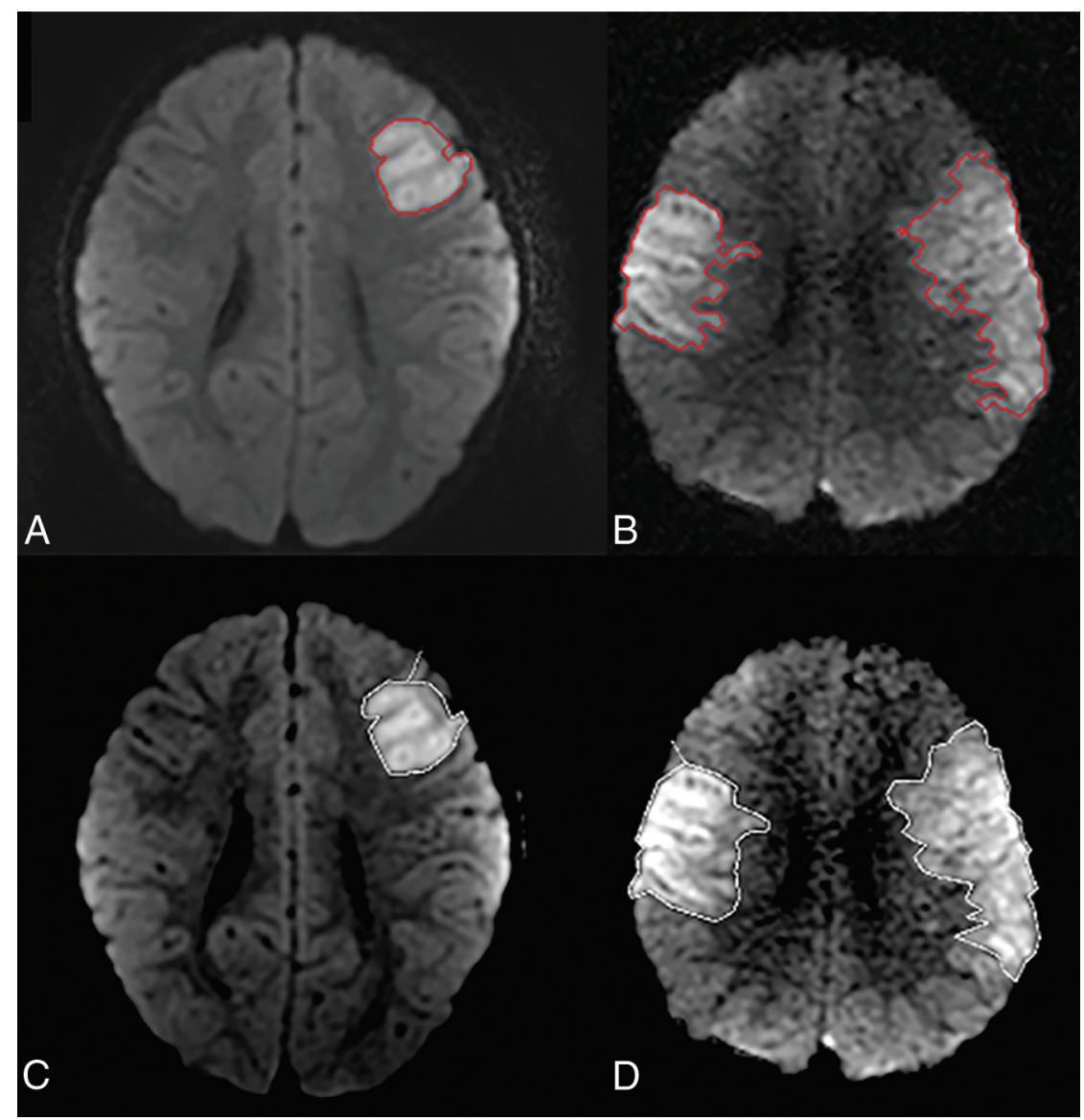

FIG 3. $A$ and $B, C A V$ segmentation results from patients in good and poor outcome groups, respectively. $C$ and $D$, Manual segmentation results from the same respective patients. lation between the methods was 0.980 (95\% CI, 0.956-0.991). The concordance correlation for CAV measurements between 2 users was 0.968 ( $95 \%$ CI, 0.935-0.985). The concordance correlation for manual segmentation between 2 users was 0.978 (95\% CI, 0.964-0.989). The concordance for mild and large infarcts with CAV measurements was 0.999 (95\% CI, 0.994-1.000) and 0.962 (95\% CI, 0.915-0.983), respectively. The concordance for mild and large infarcts with manual measurements was 0.976 (95\% CI, 0.943-0.990) and 0.976 (95\% CI, 0.953-0.983), respectively.

The mean times for segmentation between computer-assisted and manual techniques (including times for opening and saving images) were $<1$ minute and 7.3 minutes, respectively $(P<.01)$.

\section{Correlation with Clinical Outcomes}

Patient outcomes and stroke territory involvement are listed in Table 2, and patient outcomes with respect to stroke characteristics, including corresponding confidence intervals, are listed in Table 3. With regard to outcomes, $78.3 \%$ (18/23) had poor outcomes, and $21.7 \%(5 / 23)$ had good outcomes by the PSOM scores. Mortality was observed in 21.7\% (5/23) cases. Mean core infarct volumes for the good and poor outcome groups were 7.4 and $75.7 \mathrm{~mL}$, respectively $(P<.007)$. Mean percentages of infarcted brain parenchyma for the good and poor outcome groups were $0.6 \%$ and $10.4 \%$, respectively $(P<.006)$. There was a significant correlation between PSOM and infarct volume $(P<.01)$ and percentage of brain parenchymal infarction $(P<.04)$. This is displayed graphically in Fig 2 and summarized in Table 4. Core infarct volumes of $>32$ $\mathrm{mL}$ and percentage of infarcted brain parenchyma of $>3 \%$ had poor outcomes in all cases. Examples of segmentation results from CAV and manual approaches for infarcts in poor and good outcome cohorts are provided in Fig 3.

\section{DISCUSSION}

Quantification of core infarct volume within the pediatric stroke population may provide assistance in clinical decision-making and prognostic information. However, challenges in manual segmentation currently limit its utility in an acute stroke setting because it can be prohibitively time-consuming. Additionally, while the present study did not find significant variability for manual measurements, prior work has suggested that manual determination of core infarct volume is a source of variance. ${ }^{27}$ In the present study, we compared the quantification of infarct volume and the percentage of infarcted brain parenchyma by using a semiautomated, computer-assisted approach with a traditional, manual approach and observed no significant difference in 
measured infarct volumes. CAV assessment showed high correlation between users, but the CAV was significantly faster compared with the manual approach, taking seconds to perform. The rapidity of computer-assisted quantification of infarct volume allows its real-time integration into a routine, clinical workload and its use in investigative research.

The development of quantitative neuroimaging biomarkers is needed to inform treatment planning, management, and prognostication in the setting of acute ischemic infarct in children as well as clinical trials, which need objective metrics for the assessment of clinical outcomes and cost effectiveness. Several prior studies have shown a correlation between final infarct volume and worse neurologic outcome. Zecavati et $\mathrm{al}^{11}$ observed that core infarct volumes in pediatric patients, as determined on DWI, that exceed $10 \%$ of brain parenchymal volume were associated with poor neurologic outcome at 30 days; however, the authors used the Glasgow Outcome Score, which, unlike the PSOM, has not been specifically validated in the pediatric population. The PSOM is based on a standardized, neurologic examination; is currently the best validated outcome measure; and has shown excellent interobserver correlation and validity and reproducibility when used retrospectively to analyze neurologic examinations or in prospective longitudinal research. ${ }^{20,21,28}$ In our study, we assessed the correlation between core infarct volume and clinical outcome by using the Pediatric Stroke Outcome Measure and observed that both infarct volume and the percentage of infarcted brain parenchyma correlated significantly with outcome. Specifically, cutoff values above $32 \mathrm{~mL}$ for core infarct volume and 3\% for percentage of infarcted brain parenchymal volume were always associated with a bad outcome.

The CAV approach is robust; it is applicable to cases of solitary and multiple lesions and may represent an advance over qualitative vascular territory-based stroke scales. In addition, the software easily delineates total brain parenchymal volume excluding extra-axial CSF so that the percentage of infarcted brain parenchyma can be easily quantified. Beyond its utility in normalizing the infarct volume, CAV can be used independently for assessing total parenchymal or ventricular volume and may have additional applications in pediatric neuroradiology. Incorporation of CAV methods in standard clinical workflow has profound implications for the relevance of the radiologic consultation. In the context of pediatric stroke, CAV may identify children who would benefit from more aggressive therapy. CAV may quantify volumetric thresholds that could be used as end points for future clinical trials in pediatric stroke, and semiautomated methods may facilitate translation of these results from clinical trials into clinical practice.

When considering our results, several limitations should be kept in mind. First, we conducted a retrospective study, and our cohort of 23 patients is modest in size, which limits evaluation for confounders. Second, inclusion of neonates could be an additional confounder because longer follow-up may be needed because there may be a delay in the appearance of deficits after neonatal stroke, given that subtle sequelae may be difficult to delineate on neurologic examination in an incompletely myelinated, immature brain. ${ }^{20,28}$ However, the average age in our study was almost 5 years. Because our study was based at a tertiary referral center, the poor outcome category could have been over- represented. Children with associated neurologic disorders are included because stroke occurs with increased incidence in this group and gives the results wider applicability, and other investigators have validated the inclusion of children with neurologic disorders. ${ }^{28}$ Additionally, our study is based on calculation of infarct volume on DWI sequences in the acute setting within 72 hours of diagnosis. However, Ebinger et $\mathrm{al}^{12}$ have shown that subacute reduced diffusion volume in adults tightly correlated with final neurologic outcome assessed with the National Institutes of Health Stroke Scale, but most studies have shown stable DWI lesion evolution from 36 hours to 2 weeks. ${ }^{12,29}$

\section{CONCLUSIONS}

CAV quantification of core infarct volume in acute arterial ischemic infarct in pediatric patients provides a reproducible and significantly faster result comparable with manual techniques, which allows its easy integration into routine, clinical workflow. In our study, cutoff values above $32 \mathrm{~mL}$ for core infarct volume and 3\% for percentage of infarcted brain parenchymal volume were associated with a poor outcome in all patients.

Disclosures: Christopher G. Filippi-RELATED: Grant: Radiological Society of North America Medical Student Seed Grant, ${ }^{*}$ Comments: At the time of the study, coauthor Alexander El-Ali was a medical student; he is now a doctor, having graduated in May; UNRELATED: Consultancy: Regeneron Pharmaceuticals, Syntactx; Grants/ Grants Pending: National Institutes of Health, Comments: 7/01/2012 to 4/30/2017; "Chronic Convection-Enhanced Delivery of Topotecan in Malignant Glioblastoma Multiforme"; Collaborator: C.G. Filippi (10\% effort); Principal Investigator: Jeffrey Bruce; National Institutes of Health/National Cancer Institute; 1R01CA161404-01A; Other: advisor to resident and medical student as a research mentor, ${ }^{*}$ Comments: Medical student and radiology residents have Radiological Society of North America grants for which I am scientific mentor, but this is unrelated to this work because it deals with $\mathrm{Tl} \rho \mathrm{MR}$ imaging. *Money paid to the institution.

\section{REFERENCES}

1. deVeber G, Roach ES, Riela AR, et al. Stroke in children: recognition, treatment, and future directions. Semin Pediatr Neurol 2000;7:309-17

2. Carandang R, Seshadri S, Beiser A, et al. Trends in incidence, lifetime risk, severity, and 30-day mortality of stroke over the past 50 years. JAMA 2006;296:2939-46

3. Kissela BM, Khoury JC, Alwell K, et al. Age at stroke: temporal trends in stroke incidence in a large, biracial population. Neurology 2012;79:1781-87

4. George MG, Tong X, Kuklina EV, et al. Trends in stroke hospitalizations and associated risk factors among children and young adults, 1995-2008. Ann Neurol 2011;70:713-21

5. Fox CK, Fullerton HJ. Recent advances in childhood arterial ischemic stroke. Curr Atheroscler Rep 2010;12:217-24

6. Sultan S, Elkind MS. Stroke in young adults: on the rise? Neurology 2012;79:1752-53

7. Gardner MA, Hills NK, Sidney S, et al. The 5-year direct medical cost of neonatal and childhood stroke in a population-based cohort. Neurology 2010;74:372-78

8. Simma B, Holiner I, Luetschg J. Therapy in pediatric stroke. Eur J Pediatr 2013;172:867-75

9. Engelmann KA, Jordan LC. Outcome measures used in pediatric stroke studies: a systematic review. Arch Neurol 2012;69:23-27

10. Lequin MH, Dudink J, Tong KA, et al. Magnetic resonance imaging in neonatal stroke. Semin Fetal Neonatal Med 2009;14:299-310

11. Zecavati N, Singh R, Farias-Moeller R, et al. The utility of infarct volume measurement in pediatric ischemic stroke. J Child Neurol 2013;29:811-17

12. Ebinger M, Christensen S, De Silva DA, et al. Expediting MRI-based 
proof-of-concept stroke trials using an earlier imaging end point. Stroke 2009;40:1353-58

13. Yoo AJ, Chaudhry ZA, Nogueira RG, et al. Infarct volume is a pivotal biomarker after intra-arterial stroke therapy. Stroke 2012;43: 1323-30

14. Ganesan V, Ng V, Chong WK, et al. Lesion volume, lesion location, and outcome after middle cerebral artery territory stroke. Arch Dis Child 1999;81:295-300

15. Domi T, deVeber G, Shroff M, et al. Corticospinal tract pre-Wallerian degeneration: a novel outcome predictor for pediatric stroke on acute MRI. Stroke 2009;40:780-87

16. Beaulieu C, de Crespigny A, Tong DC, et al. Longitudinal magnetic resonance imaging study of perfusion and diffusion in stroke: evolution of lesion volume and correlation with clinical outcome. Ann Neurol 1999;46:568-78

17. Baird AE, Lövblad KO, Dashe JF, et al. Clinical correlations of diffusion and perfusion lesion volumes in acute ischemic stroke. Cerebrovasc Dis 2000;10:441-48

18. Tong DC, Yenari MA, Albers GW, et al. Correlation of perfusionand diffusion-weighted MRI with NIHSS score in acute ( $<6.5$ hour) ischemic stroke. Neurology 1998;50:864-70

19. Lövblad KO, Baird AE, Schlaug G, et al. Ischemic lesion volumes in acute stroke by diffusion-weighted magnetic resonance imaging correlate with clinical outcome. Ann Neurol 1997;42:164-70

20. Kitchen L, Westmacott R, Friefeld S, et al. The Pediatric Stroke Outcome Measure: a validation and reliability study. Stroke 2012;43: 1602-08

21. deVeber GA, MacGregor D, Curtis R, et al. Neurologic outcome in survivors of childhood arterial ischemic stroke and sinovenous thrombosis. J Child Neurol 2000;15:316-24

22. Guo X, Zhao B. A method and apparatus for infarct segmentation. Columbia University Invention Report IR \#CU15049. http:// innovation.columbia.edu/technologies/2906_cumc-radiologyimaging-lab-available-technologies. Accessed November 24, 2014

23. Guo X, Zhao B, Schwartz LH. Methods and systems for segmentation of organs and tumors and objects. US Patent application PCT/ US13/36166. April 1, 2013

24. Guo X, Schwartz LH, and Zhao B. Semi-automated segmentation of multimodal brain tumor using active contours. In: Proceedings of NCI-MICCAI BRATS, Nagoya, Japan. September 22-26, 2013: $17-30$

25. De Marchis GM, Filippi CG, Guo X, et al. Brain injury visible on early MRI after subarachnoid hemorrhage might predict neurological impairment and functional outcome. Neurocritic Care $2014 \mathrm{Jul}$ 11. [Epub ahead of print]

26. Chow DS, Qi J, Guo X, et al. Semiautomated volumetric measurement on postcontrast MR imaging for analysis of recurrent and residual disease in glioblastoma multiforme. AJNR Am J Neuroradiol 2014;35:498-503

27. Rafay MF, Pontigon AM, Chiang J, et al. Delay to diagnosis in acute pediatric arterial ischemic stroke. Stroke 2009;40:58-64

28. deVeber G, Andrew M, Adams C, et al. Cerebral sinovenous thrombosis in children. $N$ Engl J Med 2001;345:417-23

29. Lansberg MG, O'Brien MW, Tong DC, et al. Evolution of cerebral infarct volume assessed by diffusion-weighted magnetic resonance imaging. Arch Neurol 2001;58:613-17 